\title{
Using bioinformatics and metabolomics to identify altered granulosa cells in patients with diminished ovarian reserve
}

\author{
Ruifen He ${ }^{1}$, Zhongying Zhao ${ }^{1}$, Yongxiu Yang ${ }^{2}$, Xiaolei Liang ${ }^{\text {Corresp. } 2}$ \\ ${ }^{1}$ The First Clinical Medical College of Lanzhou University, Lanzhou, China \\ China \\ Corresponding Author: Xiaolei Liang \\ Email address: liangx107@lzu.edu.cn
}

Background: During fertility treatment, diminished ovarian reserve (DOR) is a challenge that can seriously affect a patient's reproductive potential. However, the pathogenesis of DOR is still unclear and its treatment options are limited. This study aimed to explore DOR's molecular mechanisms.

Methods: We used R software to analyze the mRNA microarray dataset E-MTAB-391 downloaded from ArrayExpress, screen for differentially expressed genes (DEGs), and perform functional enrichment analyses. We also constructed the protein-protein interaction (PPI) and miRNA-mRNA networks. Ovarian granulosa cells (GCs) from women with DOR and the control group were collected to perform untargeted metabolomics analyses. Additionally, small molecule drugs were identified using the Connectivity Map database.

Results : We ultimately identified 138 DEGs. Our gene ontology (GO) analysis indicated that DEGs were mainly enriched in cytokine and steroid biosynthetic processes. According to the Kyoto Encyclopedia of Genes and Genomes (KEGG), the DEGs were mainly enriched in the AGE (advanced glycation endproduct)-RAGE (receptor for AGE) signaling pathway in diabetic complications and steroid biosynthesis. In the PPI network, we determined that JUN, EGR1, HMGCR, ATF3, and SQLE were hub genes that may be involved in steroid biosynthesis and inflammation. MiRNAs also played a role in DOR development by regulating target genes. We validated the differences in steroid metabolism across GCs using liquid chromatography-tandem mass spectrometry (LC-MS/MS). We selected 31 small molecules with potentially positive or negative influences on DOR development.

Conclusion : We found that steroidogenesis and inflammation played critical roles in DOR development, and our results provide promising insights for predicting and treating DOR. 


\section{Using bioinformatics and metabolomics to identify altered \\ 2 granulosa cells in patients with diminished ovarian reserve}

3

4

5 Ruifen $\mathrm{He}^{1}$, Zhongying Zhao ${ }^{1}$, Yongxiu Yang ${ }^{2}$, Xiaolei Liang ${ }^{2}$

6

$7{ }^{1}$ The First Clinical Medical College of Lanzhou University, Lanzhou, China

$8 \quad{ }^{2}$ Department of Obstetrics and Gynecology, The First Hospital of Lanzhou University, Key

9 Laboratory for Gynecologic Oncology Gansu Province, Lanzhou, China

11 Corresponding Author:

12 Xiaolei Liang

13

Lanzhou City, Gansu Province, China

14

Email address: liangx107@1zu.edu.cn 
20

21

22

\section{ABSTRACT}

Background: During fertility treatment, diminished ovarian reserve (DOR) is a challenge that can seriously affect a patient's reproductive potential. However, the pathogenesis of DOR is still unclear and its treatment options are limited. This study aimed to explore DOR's molecular mechanisms.

Methods: We used R software to analyze the mRNA microarray dataset E-MTAB-391 downloaded from ArrayExpress, screen for differentially expressed genes (DEGs), and perform functional enrichment analyses. We also constructed the protein-protein interaction (PPI) and miRNA-mRNA networks. Ovarian granulosa cells (GCs) from women with DOR and the control group were collected to perform untargeted metabolomics analyses. Additionally, small molecule drugs were identified using the Connectivity Map database.

Results: We ultimately identified 138 DEGs. Our gene ontology (GO) analysis indicated that DEGs were mainly enriched in cytokine and steroid biosynthetic processes. According to the Kyoto Encyclopedia of Genes and Genomes (KEGG), the DEGs were mainly enriched in the AGE (advanced glycation end-product)-RAGE (receptor for AGE) signaling pathway in diabetic complications and steroid biosynthesis. In the PPI network, we determined that JUN, EGR1, HMGCR, ATF3, and SQLE were hub genes that may be involved in steroid biosynthesis and inflammation. MiRNAs also played a role in DOR development by regulating target genes. We validated the differences in steroid metabolism across GCs using liquid chromatography-tandem mass spectrometry (LC-MS/MS). We selected 31 small molecules with potentially positive or negative influences on DOR development. 
41 Conclusion: We found that steroidogenesis and inflammation played critical roles in DOR

42 development, and our results provide promising insights for predicting and treating DOR.

\section{INTRODUCTION}

44 Diminished ovarian reserve (DOR), defined as a decline in the number or quality of follicles and oocytes, reduces a female patient's reproductive potential (Sharara et al., 1998). The incidence of DOR ranges from $9 \%$ to $24 \%$ in women undergoing in vitro fertilization (IVF) (Kyrou et al., 2009). The most common effective treatment for DOR is the use of assisted reproductive techniques. Ovarian reserve naturally declines with age, but some women experience DOR much earlier than average. Young women with DOR thus have an accelerated physiological decline in ovarian reserve. DOR is one of the greatest challenges for reproductive endocrinologists because it is characterized by poor ovarian response to gonadotrophin stimulation, low pregnancy rates, and high rates of pregnancy loss (Levi et al., 2001; Navot et al., 1987).

An ovarian follicle is a complex structure comprised of oocytes, cumulus cells (CCs), and granulosa cells (GCs). The bidirectional communication between the oocyte and its companion somatic cells is essential for follicular development and oocyte growth (Anderson \& Albertini 1976; Matzuk et al., 2002). In mice, the oocyte's transcriptional activity was modulated when in vitro cultured with GCs, but not without GCs (De La Fuente \& Eppig 2001). Another study showed that DOR patients had an increase in GC apoptosis, which is associated with a poor ovarian response and oocyte yield (Fan et al., 2019). Considering this coadjutant relationship between oocyte and GC, exploring the alteration of GCs from women with DOR may provide a deeper understanding for DOR pathogenesis. Previous studies have investigated the mRNA expression profiles of ovarian CCs (Greenseid et al., 2011) and GCs (Chin et al., 2002; Skiadas 
63 et al., 2012) in addition to miRNA expression patterns (Chen et al., 2017; Woo et al., 2018) in

64 DOR patients. These studies typically had different inclusion criteria for DOR patients. MiRNAs

65 are small noncoding RNAs that play critical roles in many diseases and biological processes,

66 including reproduction, and regulate mRNA translation and stability (Fabian et al., 2010).

67 Exploring how miRNA affects female reproduction (Sabry et al., 2019) could reveal miRNA

68 therapeutics as a possible DOR treatment option.

69 Steroid hormones are a type of steroid involved in many biological and physiological

70 functions. Cholesterol is the precursor for steroid hormone synthesis (Greaves et al., 2014).

71 Steroid hormone levels affect the follicular growth and development processes (Chou \& Chen

72 2018). Inflammation triggers a wide range of physiological and pathological processes

73 (Medzhitov 2008). Aberrant inflammation has a negative effect on folliculogenesis and

ovulation, and polycystic ovary syndrome (PCOS) is associated with the chronic endogenous production of low-grade pro-inflammatory cytokines (Boots \& Jungheim 2015). Inflammation and steroidogenesis abnormalities may also be involved in the development of DOR.

7 In this study, we performed bioinformatic analyses on the mRNA expression profiles of DOR GCs from the publicly available dataset E-MTAB-391 in order to identify differentially expressed genes (DEGs). We constructed protein-protein interaction (PPI) networks based on the DEGs, and a miRNA-mRNA network using the differentially expressed miRNAs (DEMs) extracted from a previous study (Woo et al., 2018). Small molecule drugs with potential synergistic or antagonistic effects on DOR were also screened using the Connectivity Map (CMap) database (Lamb et al., 2006). Moreover, we applied liquid chromatography-tandem mass spectrometry (LC-MS/MS) on our samples to explore the metabolic alteration of GCs. This study may shed light on the future of DOR prognosis and treatment. 


\section{MATERIALS \& METHODS}

87

88

89

90

91

92

\section{Data collection}

We screened GEO and ArrayExpress (https://www.ebi.ac.uk/arrayexpress/) for expressed profiles of GCs from patients with and without DOR. We excluded datasets that: (1) were without detailed sample information, (2) used samples based on cell lines or animal models, (3) had sample sizes $<10$, and (4) had quite different definitions of DOR or used different participant age ranges than our study. Only one mRNA microarray dataset, E-MTAB-391 (which included 13 DOR samples and 13 normal ovarian reserve (NOR) samples (Skiadas et al., 2012)), met our criteria and was downloaded from ArrayExpress. E-MTAB-391's platform was the AMEXP-1564-IIIumina HumanRef-8 WG-DASL v3 Expression BeadChip. We directly extracted the data from the DOR miRNAs used in a previous study (Woo et al., 2018).

\section{Identifying DEGs}

We used the limma package in R software (Ritchie et al., 2015) to identify DEGs across the DOR and NOR samples. The adjusted P-value (false discovery rate) was obtained using the Benjamini-Hochberg algorithm when screening the DEGs. We set $\mathrm{P}<0.05$ and $\mid \log 2$ (fold change; FC) $\mid>0.58$ as the DEG cut-off criteria. The DEGs were divided into upregulated and downregulated DEGs and saved for subsequent analyses.

\section{Functional and enrichment analyses of DEGs}

Gene ontology (GO) annotation is widely used to study the biological functions of multiple genes, and is comprised of three independent ontologies: biological process (BP), molecular function (MF), and cellular component (CC) (Ashburner et al., 2000). The Kyoto Encyclopedia of Genes and Genomes (KEGG) pathway analysis is a valuable tool used to assess the interaction 
108 networks of genes and their products (Kanehisa et al., 2017). In this study, we used the

109 clusterProfiler package in R software to obtain the GO and KEGG pathway enrichment for the

110 DEGs (Yu et al., 2012). We set the value of $\mathrm{P}<0.05$ as the threshold for significance.

\section{PPI network construction and module analysis}

112 The STRING database offers PPI assessment and integration (Szklarczyk et al., 2017). To

113 achieve a system-wide understanding across DEGs, we constructed the PPI network using

114 STRING (version 10.5; https://string-db.org/cgi/input.pl) with a combined cutoff score $\geq 4$. The

115 network was visualized using Cytoscape (version 3.6.1; https://cytoscape.org/), which can

116 integrate biomolecular interaction networks into a unified conceptual framework (Shannon et al.,

117 2003). The nodes and edges of the network represent proteins and protein-protein associations,

118 respectively. We performed a module analysis of the PPI network based on the Molecular

119 Complex Detection (MCODE) feature of the Cytoscape software using the following parameters:

120 degree cut-off $=2$, node score cut-off $=0.2$, $\max$ depth $=100$, and $\mathrm{k}$-score $=2$. We performed

121 subsequent GO and KEGG pathway analyses of the selected modules using the clusterProfiler

122 package.

\section{Exploring DEM target genes}

124 We extracted 105 DEMs from the GCs of women diagnosed with DOR in a previous study (Woo

125 et al., 2018). The multiMiR package integrated 11 miRNA-target databases (three validated and eight predicted miRNA-target databases) and three disease-/drug-related miRNA databases (Ru

127 et al., 2014) to retrieve interactions between the DEMs and screened DEGs. The DEM target

128 genes were only screened from the 11 miRNA-target databases. Finally, we visualized the

129 regulatory miRNA-mRNA network using Cytoscape software. 


\section{Identifying small molecules}

131 The CMap database (https://portals.broadinstitute.org/cmap) contains 7,000 gene-expression

132 profiles from cultured human cells treated with bioactive small molecules (Lamb et al., 2006),

133 and is a valuable resource when looking for connections between diseases, genetic perturbation,

134 and drug action. We mapped the upregulated and downregulated genes to the CMap database to

135 identify potential small molecule drugs, which either have antagonistic or synergistic effects on

136 DOR. We regarded $\mathrm{n}$ (the number of instances) $\geq 4$, enrichment $>0.7$, and P-value $<0.01$ as

137 statistically significant.

\section{Collecting GCs}

139 This study was approved by the ethics committee of the First Hospital of Lanzhou University 140 (LDYYLL2019-44), and we obtained written informed consent from all participants. Ovarian

141 GCs were collected from women with DOR $(n=3)$ and women with NOR $(n=3)$. All participants

142 were $\leq 35$ years old to eliminate age as a potential confounding variable. DOR was identified 143 using FSH levels $(12 \leq \mathrm{FSH}<25)$ and ovarian response (the number of follicles on the day of

144 the ovulatory human chorion gonadotropin (hCG) trigger injection $\leq 7)$. We selected infertile 145 women undergoing IVF due to male or tubal factor infertility for the NOR group. Controlled 146 ovarian stimulation was performed and follicular development was monitored using a

147 transvaginal ultrasound. Oocyte retrieval was performed 36 hours after hCG administration and 148 GCs were isolated from fluid aspirates using previously-described methods (Vanacker et al., 149 2011).

\section{LC-MS/MS experiments}


151 We performed metabolite extraction and LC-MS/MS analysis at Beijing Genomics Institute

152 (BGI). Each frozen GCs sample was thawed and weighed into $1.5 \mathrm{~mL}$ Eppendorf tubes. We

153 added the internal standard solution and $800 \mu \mathrm{L}$ of methanol/acetonitrile/water solvent $(2: 2: 1$,

$154 \mathrm{v} / \mathrm{v} / \mathrm{v}$ ) to homogenize. Mixtures were centrifuged at 25,000 rcf for $15 \mathrm{~min}$, and the supernatant

155 was transferred out and vacuum dried. We then re-extracted the metabolite extract in $200 \mu \mathrm{L}$ of a

156 methanol/water mixture (1:9, v/v). After vortexing, the samples were centrifuged again. We

157 collected the supernatant and inspected each sample using $20 \mu \mathrm{L}$ of supernatant, the Waters 2D

158 UPLC (Waters, Milford, MA, USA), and a Q Exactive high-resolution mass spectrometer

159 (Thermo Fisher Scientific, Waltham, MA, USA). The analytical column we used was an

ACQUITY UPLC BEH C18 $(1.7 \mu \mathrm{m}, 2.1 \times 100 \mathrm{~mm}$, Waters $)$. In positive ion mode, the mobile

161 phase was MS-grade water with $0.1 \%$ formic acid (A) and $100 \%$ methanol with $0.1 \%$ formic

162 acid (B). In negative ion mode, the mobile phase was MS-grade water with $10 \mathrm{mM}$ of

163 ammonium formate (A) and 95\% methanol with $10 \mathrm{mM}$ of ammonium formate (B). The extracts

164 were gradient-eluted with a flow rate of $0.35 \mathrm{ml} / \mathrm{min}$. The full scan and fragment acquisition

165 resolutions were 70,000 and 17,500, respectively. The ESI parameters were set as follows:

166 sheath gas flow rate of $40 \mathrm{~L} \cdot \mathrm{min}-1$, auxiliary gas flow rate of $10 \mathrm{~L} \cdot \mathrm{min}-1$, spray voltage of 3800

$167 \mathrm{~V}$ (positive mode) and $3200 \mathrm{~V}$ (negative mode), capillary temperature of $320^{\circ} \mathrm{C}$, and auxiliary

168 gas heater temperature of $350^{\circ} \mathrm{C}$. The LC-MS/MS data were processed using Compound

169 Discoverer 3.0 software (Thermo Fisher Scientific). We identified the differential metabolites

170 using a combination of principal component analysis (PCA) and univariate analysis.

\section{RESULTS}

\section{Identifying DEGs related to DOR}


173 In this study, we downloaded the normalized expression data from the E-MTAB-391 dataset

174 (Fig. S1). A total of 18,128 genes were available for further DEG identification. Using our

175 criteria, we selected 138 DEGs from the DOR and NOR samples, including 55 upregulated and

17683 downregulated genes. The volcano plot in Fig. 1 shows the distribution of all screened genes.

177 The heat map of all DEGs based on unsupervised hierarchical clustering is shown in Fig. S2.

\section{GO and KEGG pathway enrichment analyses of DEGs}

179 According to the GO BP analysis, the upregulated DEGs were mainly enriched in skeletal 180 muscle cell differentiation and regulation of transcription from RNA polymerase II promoter in 181 response to stress. Downregulated DEGs were mainly enriched in the steroid biosynthetic and 182 cholesterol biosynthetic processes. Fig. 2 shows the top 20 GO BP up- and down-regulated DEG terms in detail. Additionally, we performed KEGG pathway enrichment analysis and the results can be found in Table 1. The upregulated DEGs were significantly enriched in the AGE (advanced glycation end-product)-RAGE (receptor for AGE) signaling pathway in diabetic complications and human T-cell leukemia virus 1 infection, and the downregulated DEGs were mainly enriched in steroid biosynthesis (Fig. 3) and terpenoid backbone biosynthesis (Fig. S3).

\section{Constructing PPI networks and module analysis}

We imported all DEGs related to DOR into the STRING database when constructing the PPI network, which included 99 nodes and 294 edges when the cut-off combined score was set at $\geq$ node degrees were Jun proto-oncogene, AP-1 transcription factor subunit (JUN, degree=24); early growth response 1 (EGR1, degree=18); 3-hydroxy-3-methylglutaryl-CoA reductase

194 (HMGCR, degree=17); activating transcription factor 3 (ATF3, degree=15); and squalene 
195 epoxidase (SQLE, degree=15). Additionally, we filtered out the top two modules from the PPI

196 network in order to implement further GO and KEGG pathway analyses. Module 1 (Fig. 4B)

197 contained 12 downregulated genes enriched in the cholesterol biosynthetic process of the GO BP

198 term. Module 2 (Fig. 4C) contained 10 upregulated genes that were mainly enriched in skeletal

199 muscle cell differentiation of the GO BP term. The detailed results of the modules' GO and

200 KEGG pathway analyses are shown in Table 2.

201

202

203

204

205

206

207

208

209

210

211

212

213

214

215

216

\section{Predicting DEM target genes and constructing the DEM-DEG regulatory network}

We analyzed the DEMs associated with DOR, including 85 upregulated and 20 downregulated genes, using the multiMiR package to predict their target genes. We then used the identified DEM-DEG pairs (comprised of 91 DEMs and 109 DEGs) to construct the regulatory network. Among the pairs, miR-155-5p, miR-16-5p, let-7b-5p, miR-107, and miR-103a-3p had the most target genes. The detailed interactions between the DEMs and DEGs are shown in Fig. 5.

\section{Screening small molecule drugs}

In order to screen out small molecule drugs, we compared all DEGs to the gene expression profiles in CMap. We identified 31 small molecules, seven of which had negative scores with the potential to reverse DOR. The detailed results are shown in Fig. 6.

\section{Metabolic differences between DOR and NOR GCs}

Metabolites are essential for cellular function and untargeted metabolomics analyses can provide information on their associations with diseases. We analyzed the GC samples from the DOR and NOR groups using LC-MS/MS in both positive and negative ion modes. After data processing and metabolite identification, we screened the differential metabolites using a threshold p-value $<0.05$ and $\mathrm{FC} \geq 1.2$ or $\leq 0.83$. We did detect metabolic differences between the GCs of the DOR 
217 and NOR samples. The detailed differences in the steroids and metabolites observed in the GCs

218 of the two groups are listed in Table 3.

\section{DISCUSSION}

220 A patient with DOR has a reduced number of retrieved oocytes compared to other women of a

221 similar age. In some women, DOR can progress to a diagnosis of primary ovarian insufficiency

222 (POI), which is an extreme form of ovarian dysfunction (Cooper et al., 2011). Because of this

223 serious threat to a patient's reproductive health, there is an urgent need to further examine DOR

224 etiology. Previous studies have used the GC mRNA/miRNA expression profiles from DOR

225 patients to explore the molecular mechanisms of DOR. In this study, we focused on altered GCs

226 from young women with DOR, and performed LC-MS/MS experiments and bioinformatic

227 analyses to explore the differences between DOR and NOR. We obtained raw mRNA expression

228 patterns and DEM data from previous publications (Skiadas et al., 2012) (Woo et al., 2018) with

229 similar inclusion criteria to ours. The inclusion criteria are presented in Table S1.

230 Sex steroid hormones (progestogens, androgens, and estrogens) have a steroid nucleus

231 structure and are typically synthesized from cholesterol in the gonads and adrenal glands

232 (Greaves et al., 2014). These hormones play important roles in female reproduction. The

233 synthesis and secretion of estrogen are promoted by the elevated FSH levels found in patients

234 with DOR (Practice Committee of the American Society for Reproductive Medicine, 2015).

235 However, whether there is a difference in the estrogen levels of patients with DOR and NOR

236 remains controversial. In our study, we found that downregulated DEGs were mainly enriched in

237 the steroid biosynthetic process of the GO BP term (Fig. 2A). KEGG pathway analysis (Table 1)

238 showed that downregulated DEGs were mainly enriched in steroid biosynthesis and terpenoid 
239 backbone biosynthesis. Therefore, a range of steroidogenesis substances may play a major role in

240 DOR development. Consistent with our PPI network results (Fig. 4), we found several key genes

241 in the top 20 (including HMGCR, SQLE, CYP51A, HMGCS1, FDFTI, SC5D, NSDHL, IDI1,

242 EBP, and MSMO1) in steroid biosynthesis and terpenoid backbone biosynthesis pathways (Figs.

2433 and S3). All these genes were downregulated in patients with DOR from our dataset. Previous

244 studies have found that HMGCR catalyzes the first rate-limiting step in cholesterol synthesis

245 (Howe et al., 2017); HMGCS1 condenses acetyl-CoA to form 3-hydroxy-3-methylglutaryl CoA,

246 which is the substrate for HMGCR (Mathews et al., 2014); and CYP51A also participates in

247 cholesterol synthesis, which can catalyze the removal of the $14 \alpha$-methyl group from lanosterol

248 (Sharpe \& Brown 2013). Upstream biological disruptions lead to a series of metabolomic

249 changes. According to our untargeted metabolomics analysis, several steroids (Table 3) were

250 significantly lower in GCs from patients with DOR compared to the control group. Among these

251 steroids, progesterone plays an essential role in female reproductive events (ovulation,

252 implantation, and pregnancy maintenance) and serves as an intermediate during estrogen

253 biosynthesis (Gellersen et al., 2009). Prior evidence suggests that GCs can directly produce

254 progesterone before entering theca cells to convert into androgens (Oktem et al., 2017).

255 Hydroxyprogesterone acts as an intermediate during the conversion of progesterone to androgens

256 that are transported into GCs and converted into estrogen. The relationships between steroids and

257 ovarian function (Table 3) have been rarely reported on and require more study. It has been

258 demonstrated that several steroidogenic gene disturbances induced by bisphenol A can cause

259 developmental impairments of ovary tissue (Liu et al., 2019). Overall, patients with DOR may

260 have impaired hormone synthesis, which could be compensated for by elevated FSH levels. The

261 perturbation of steroidogenic genes may be responsible for DOR development. 
262 Aberrant inflammation has been suggested to influence follicular growth and development

263 (Boots \& Jungheim 2015). Our results showed that upregulated genes were enriched in the AGE-

264 RAGE signaling pathway (Table 1). The AGE-RAGE signaling pathway has been shown to

265 induce reactive oxygen species (ROS) burst and inflammation, eventually leading to POI (Huang

266 et al., 2019). EGR1 plays a proinflammatory role in numerous pathological processes and human

267 diseases (Schmidt et al., 2008). A recent study found that in mice, EGR1 was increased in aged

268 ovaries compared to young ovaries (Yuan et al., 2016). Our results showed that EGR1 as a key

269 gene in the PPI network was upregulated in women with DOR. During cholestasis, EGR1

270 regulates the production of inflammatory mediators, including cytokines and adhesion

271 molecules, that promote the accumulation and activation of inflammatory cells, causing liver

272 injuries (Bonetti et al., 2010). According to the results of our GO analysis of upregulated DEGs

273 (Fig. 2B), cytokines may be associated with DOR development. Cytokines play a key role in

274 inflammation, and can be found in the immune cells of the ovary (Tabibzadeh 1994; Vinatier et

275 al., 1995). Accumulated evidence suggests that inflammation is closely related to ovarian

276 dysfunction. Women diagnosed with PCOS often present with chronic low-grade inflammation

277 due to overactive interleukin-1 (IL-1), a proinflammatory cytokine (Popovic et al., 2019).

278 Additionally, multiple autoimmune diseases have adverse effects on female fertility via

279 premature DOR (Sen et al., 2014). Therefore, anti-inflammatory treatment may be able to

280 alleviate the progression of DOR. In a POI rat model, resveratrol counteracted inflammatory

281 signaling induced by ionizing radiation, and preserved the entire ovarian follicle pool (Said et al.,

282 2016). More studies are needed to confirm the role of inflammation in DOR development and

283 whether controlling inflammation is an option for DOR treatment. 
MiRNAs, a class of endogenous non-coding small molecule RNA, play an important role in

285

286

287

288

289

290

291

292

293

294

295

296

297

298

299

300

301

302

303

304

305

306

gene expression modulation at the post-transcriptional level. Previous studies have shown that miRNAs help regulate reproductive functions, particularly follicular development, oocyte maturation, corpus function, pregnancy establishment, and early embryonic development (Eisenberg et al., 2015; Tesfaye et al., 2016). The role of miRNAs in ovarian function has been demonstrated primarily by the conditional knockout of Dicer (Luense et al., 2009), a cytoplasmic RNase III required for miRNA production in mammals. In a mouse model, the conditional knockout of Dicer in ovarian GCs led to decreased ovulation rates (Nagaraja et al., 2008) and compromised folliculogenesis and POI in oocytes (Yuan et al., 2014). In our study, we found that steroidogenic genes were regulated by differentially expressed miRNAs. HMGCS1 was regulated by 25 DEMs, including miR-155-5p, miR-16-5p, let-7b-5p, miR-107, and miR-103a3p. Furthermore, a single miRNA can target multiple genes. MiR-107 targets 21 DEGs, including five steroidogenic genes: HMGCS1, FDFT1, CYP51A1, SQLE, and EBP. miRNA-107 expression in murine ovarian GCs exposed to cadmium was significantly different from expression in the control group, and miRNA-107 can regulate kit ligand (kitl) expression (Wang et al., 2018). Kitl plays an important role in the recruitment of primitive follicles (Parrott \& Skinner 1999), the proliferation and differentiation of GCs, the recruitment of theca cells, and early steroid hormone synthesis (Flanagan et al., 1991). Therefore, miRNAs may contribute to DOR development by regulating target genes. microRNA therapies for several diseases have reached clinical testing stages with promising results (Rupaimoole \& Slack 2017). miRNAs should be researched for potential DOR treatments.

$$
\text { We also conducted CMap analysis to quickly identify molecule drugs with antagonistic or }
$$
synergistic effects on DOR based on their gene expression profiles. We found seven agents with 
307 negative scores that had potential for DOR treatment (Fig. 6). Among these, H-7 (1-(5-

308 isoquinolinesulfonyl)-2-methylpiperazine), an inhibitor of protein kinase $\mathrm{C}$, has been found to

309 reduce the release of oocytes from rat ovaries (Shimamoto et al., 1993). Estriol is a form of

310 estrogen, and a meta-analysis concluded that luteal estradiol stimulation in assisted reproductive

311 technology could decrease cycle cancellation rates and increase clinical pregnancy rates in poor

312 responders exposed to controlled ovarian hyperstimulation (Reynolds et al., 2013). Therefore, we

313 hypothesized that these molecule drugs, identified by bioinformatic analysis, may provide novel

314 DOR treatment. Further validation of their effects is still needed.

315 Despite our study's promising findings, there were still limitations. We identified possible

316 DEGs using $|\log 2 \mathrm{FC}|>0.58$ (the approximate fold change was $>1.5$ ), which is a relatively lower

317 criterion than those used by other studies. DOR is an early stage ovarian reserve impairment that

318 may take several years to develop into POI, and subtle alterations may have broader significance

319 during its development. Small gene expression changes are also worth noting. In addition, we

320 derived the E-MTAB-391 data from a large sample size. Nevertheless, we validated the steroid

321 metabolism differences between DOR and NOR samples using LC-MS/MS. Therefore, our

322 findings are reliable and provide valuable insight into DOR development.

323 CONCLUSION

324 We used bioinformatics approaches to investigate the perturbed steroidogenic and inflammation-

325 related genes that may be regulated by miRNAs in women with DOR. Using metabolomics, we

326 found that steroid metabolites were reduced in the GCs from DOR samples. Additionally, several

327 small molecule drugs (e.g., the steroid hormone estriol) with potential antagonistic or synergistic

328 effects on DOR were screened out. Our results suggest that steroidogenesis and inflammation 
329

330

331

332

333

334

335

336

337

338

339

340

341

342

343

344

345

346

347

348

349

350

351

352

353

354

355

356

357

358

359

360

361

362

363

364

365

366

367

368 play critical roles in DOR development, and should be pursued in future studies on DOR

prediction and treatment.

\section{REFERENCES}

Practice Committee of the American Society for Reproductive Medicine, 2015. Testing and interpreting measures of ovarian reserve: a committee opinion. Fertil Steril 103:e9-e17. 10.1016/j.fertnstert.2014.12.093

Anderson E, and Albertini DF. 1976. Gap junctions between the oocyte and companion follicle cells in the mammalian ovary. J Cell Biol 71:680-686. 10.1083/jcb.71.2.680

Ashburner M, Ball CA, Blake JA, Botstein D, Butler H, Cherry JM, Davis AP, Dolinski K, Dwight SS, Eppig JT, Harris MA, Hill DP, Issel-Tarver L, Kasarskis A, Lewis S, Matese JC, Richardson JE, Ringwald M, Rubin GM, and Sherlock G. 2000. Gene ontology: tool for the unification of biology. The Gene Ontology Consortium. Nat Genet 25:25-29. 10.1038/75556

Bonetti TC, Salomao R, Brunialti M, Braga DP, Borges E, Jr., and Silva ID. 2010. Cytokine and hormonal profile in serum samples of patients undergoing controlled ovarian stimulation: interleukin1beta predicts ongoing pregnancy. Hum Reprod 25:2101-2106. 10.1093/humrep/deq171

Boots CE, and Jungheim ES. 2015. Inflammation and Human Ovarian Follicular Dynamics. Semin Reprod Med 33:270-275. 10.1055/s-0035-1554928

Chen D, Zhang Z, Chen B, Ji D, Hao Y, Zhou P, Wei Z, and Cao Y. 2017. Altered microRNA and Piwiinteracting RNA profiles in cumulus cells from patients with diminished ovarian reserve. Biol Reprod 97:91-103. 10.1093/biolre/iox062

Chin KV, Seifer DB, Feng B, Lin Y, and Shih WC. 2002. DNA microarray analysis of the expression profiles of luteinized granulosa cells as a function of ovarian reserve. Fertil Steril 77:1214-1218. 10.1016/s0015-0282(02)03114-x

Chou CH, and Chen MJ. 2018. The Effect of Steroid Hormones on Ovarian Follicle Development. Vitam Horm 107:155-175. 10.1016/bs.vh.2018.01.013

Cooper AR, Baker VL, Sterling EW, Ryan ME, Woodruff TK, and Nelson LM. 2011. The time is now for a new approach to primary ovarian insufficiency. Fertil Steril 95:1890-1897. 10.1016/j.fertnstert.2010.01.016

De La Fuente R, and Eppig JJ. 2001. Transcriptional activity of the mouse oocyte genome: companion granulosa cells modulate transcription and chromatin remodeling. Dev Biol 229:224-236. 10.1006/dbio.2000.9947

Eisenberg I, Kotaja N, Goldman-Wohl D, and Imbar T. 2015. microRNA in Human Reproduction. Advances in experimental medicine and biology 888:353-387. 10.1007/978-3-319-22671-2_18

Fabian MR, Sonenberg N, and Filipowicz W. 2010. Regulation of mRNA translation and stability by microRNAs. Annu Rev Biochem 79:351-379. 10.1146/annurev-biochem-060308-103103

Fan Y, Chang Y, Wei L, Chen J, Li J, Goldsmith S, and Silber S. 2019. Apoptosis of mural granulosa cells is increased in women with diminished ovarian reserve. 36:1225-1235. 10.1007/s10815-01901446-5 
369

370

371

372

373

374

375

376

377

378

379

380

381

382

383

384

385

386

387

388

389

390

391

392

393

394

395

396

397

398

399

400

401

402

403

404

405

406

407

408

409

410

411

412

413

414

415

Flanagan JG, Chan DC, and Leder P. 1991. Transmembrane form of the kit ligand growth factor is determined by alternative splicing and is missing in the Sld mutant. Cell 64:1025-1035. 10.1016/0092-8674(91)90326-t

Gellersen B, Fernandes MS, and Brosens JJ. 2009. Non-genomic progesterone actions in female reproduction. Hum Reprod Update 15:119-138. 10.1093/humupd/dmn044

Greaves RF, Jevalikar G, Hewitt JK, and Zacharin MR. 2014. A guide to understanding the steroid pathway: new insights and diagnostic implications. Clin Biochem 47:5-15. 10.1016/j.clinbiochem.2014.07.017

Greenseid K, Jindal S, Hurwitz J, Santoro N, and Pal L. 2011. Differential granulosa cell gene expression in young women with diminished ovarian reserve. Reprod Sci 18:892-899. 10.1177/1933719111398502

Howe V, Sharpe L, Prabhu AV, and Brown AJ. 2017. New insights into cellular cholesterol acquisition: promoter analysis of human HMGCR and SQLE, two key control enzymes in cholesterol synthesis. Biochim Biophys Acta Mol Cell Biol Lipids 1862:647-657. 10.1016/j.bbalip.2017.03.009

Huang Y, Hu C, Ye H, Luo R, Fu X, Li X, Huang J, Chen W, and Zheng Y. 2019. Inflamm-Aging: A New Mechanism Affecting Premature Ovarian Insufficiency. J Immunol Res 2019:8069898. 10.1155/2019/8069898

Kanehisa M, Furumichi M, Tanabe M, Sato Y, and Morishima K. 2017. KEGG: new perspectives on genomes, pathways, diseases and drugs. Nucleic Acids Res 45:D353-d361. 10.1093/nar/gkw1092

Kyrou D, Kolibianakis EM, Venetis CA, Papanikolaou EG, Bontis J, and Tarlatzis BC. 2009. How to improve the probability of pregnancy in poor responders undergoing in vitro fertilization: a systematic review and meta-analysis. Fertil Steril 91:749-766. 10.1016/j.fertnstert.2007.12.077

Lamb J, Crawford ED, Peck D, Modell JW, Blat IC, Wrobel MJ, Lerner J, Brunet JP, Subramanian A, Ross KN, Reich M, Hieronymus H, Wei G, Armstrong SA, Haggarty SJ, Clemons PA, Wei R, Carr SA, Lander ES, and Golub TR. 2006. The Connectivity Map: using gene-expression signatures to connect small molecules, genes, and disease. Science 313:1929-1935. 10.1126/science.1132939

Levi AJ, Raynault MF, Bergh PA, Drews MR, Miller BT, and Scott RT, Jr. 2001. Reproductive outcome in patients with diminished ovarian reserve. Fertil Steril 76:666-669. 10.1016/s00150282(01)02017-9

Liu Y, Wang L, Zhu L, Ran B, and Wang Z. 2019. Bisphenol A disturbs transcription of steroidogenic genes in ovary of rare minnow Gobiocypris rarus via the abnormal DNA and histone methylation. Chemosphere 240:124935. 10.1016/j.chemosphere.2019.124935

Luense L, Carletti MZ, and Christenson LK. 2009. Role of Dicer in female fertility. Trends Endocrinol Metab 20:265-272. 10.1016/j.tem.2009.05.001

Mathews ES, Mawdsley DJ, Walker M, Hines JH, Pozzoli M, and Appel B. 2014. Mutation of 3-hydroxy-3methylglutaryl CoA synthase I reveals requirements for isoprenoid and cholesterol synthesis in oligodendrocyte migration arrest, axon wrapping, and myelin gene expression. J Neurosci 34:3402-3412. 10.1523/jneurosci.4587-13.2014

Matzuk MM, Burns KH, Viveiros MM, and Eppig JJ. 2002. Intercellular communication in the mammalian ovary: oocytes carry the conversation. Science 296:2178-2180. 10.1126/science.1071965

Medzhitov R. 2008. Origin and physiological roles of inflammation. Nature 454:428-435. 10.1038/nature07201

Nagaraja AK, Andreu-Vieyra C, Franco HL, Ma L, Chen R, Han DY, Zhu H, Agno JE, Gunaratne PH, DeMayo FJ, and Matzuk MM. 2008. Deletion of Dicer in somatic cells of the female reproductive tract causes sterility. Mol Endocrinol 22:2336-2352. 10.1210/me.2008-0142

Navot D, Rosenwaks Z, and Margalioth EJ. 1987. Prognostic assessment of female fecundity. Lancet 2:645-647. 10.1016/s0140-6736(87)92439-1

Peer] reviewing PDF | (2020:03:46424:2:0:NEW 1 Aug 2020) 
416

417

418

419

420

421

422

423

424

425

426

427

428

429

430

431

432

433

434

435

436

437

438

439

440

441

442

443

444

445

446

447

448

449

450

451

452

453

454

455

456

457

458

459

460

461

462

463

Oktem O, Akin N, Bildik G, Yakin K, Alper E, Balaban B, and Urman B. 2017. FSH Stimulation promotes progesterone synthesis and output from human granulosa cells without luteinization. Hum Reprod 32:643-652. 10.1093/humrep/dex010

Parrott JA, and Skinner MK. 1999. Kit-ligand/stem cell factor induces primordial follicle development and initiates folliculogenesis. Endocrinology 140:4262-4271. 10.1210/endo.140.9.6994

Popovic M, Sartorius G, and Christ-Crain M. 2019. Chronic low-grade inflammation in polycystic ovary syndrome: is there a (patho)-physiological role for interleukin-1? Semin Immunopathol 41:447459. 10.1007/s00281-019-00737-4

Reynolds KA, Omurtag KR, Jimenez PT, Rhee JS, Tuuli MG, and Jungheim ES. 2013. Cycle cancellation and pregnancy after luteal estradiol priming in women defined as poor responders: a systematic review and meta-analysis. Hum Reprod 28:2981-2989. 10.1093/humrep/det306

Ritchie ME, Phipson B, Wu D, Hu Y, Law CW, Shi W, and Smyth GK. 2015. limma powers differential expression analyses for RNA-sequencing and microarray studies. Nucleic Acids Res 43:e47. 10.1093/nar/gkv007

Ru Y, Kechris KJ, Tabakoff B, Hoffman P, Radcliffe RA, Bowler R, Mahaffey S, Rossi S, Calin GA, Bemis L, and Theodorescu D. 2014. The multiMiR R package and database: integration of microRNAtarget interactions along with their disease and drug associations. Nucleic Acids Res 42:e133. 10.1093/nar/gku631

Rupaimoole R, and Slack FJ. 2017. MicroRNA therapeutics: towards a new era for the management of cancer and other diseases. Nat Rev Drug Discov 16:203-222. 10.1038/nrd.2016.246

Sabry R, Yamate J, Favetta L, and LaMarre J. 2019. MicroRNAs: potential targets and agents of endocrine disruption in female reproduction. J Toxicol Pathol 32:213-221. 10.1293/tox.2019-0054

Said RS, El-Demerdash E, Nada AS, and Kamal MM. 2016. Resveratrol inhibits inflammatory signaling implicated in ionizing radiation-induced premature ovarian failure through antagonistic crosstalk between silencing information regulator 1 (SIRT1) and poly(ADP-ribose) polymerase 1 (PARP-1). Biochem Pharmacol 103:140-150. 10.1016/j.bcp.2016.01.019

Schmidt J, Stoffels B, Moore BA, Chanthaphavong RS, Mazie AR, Buchholz BM, and Bauer AJ. 2008. Proinflammatory role of leukocyte-derived Egr-1 in the development of murine postoperative ileus. Gastroenterology 135:926-936, 936.e921-922. 10.1053/j.gastro.2008.05.079

Sen A, Kushnir VA, Barad DH, and Gleicher N. 2014. Endocrine autoimmune diseases and female infertility. Nat Rev Endocrinol 10:37-50. 10.1038/nrendo.2013.212

Shannon P, Markiel A, Ozier O, Baliga NS, Wang JT, Ramage D, Amin N, Schwikowski B, and Ideker T. 2003. Cytoscape: a software environment for integrated models of biomolecular interaction networks. Genome Res 13:2498-2504. 10.1101/gr.1239303

Sharara FI, Scott RT, Jr., and Seifer DB. 1998. The detection of diminished ovarian reserve in infertile women. Am J Obstet Gynecol 179:804-812. 10.1016/s0002-9378(98)70087-0

Sharpe LJ, and Brown AJ. 2013. Controlling cholesterol synthesis beyond 3-hydroxy-3-methylglutarylCoA reductase (HMGCR). J Biol Chem 288:18707-18715. 10.1074/jbc.R113.479808

Shimamoto T, Yamoto M, and Nakano R. 1993. Possible involvement of protein kinase $C$ in gonadotropin-induced ovulation in the rat ovary. Endocrinology 133:2127-2132. 10.1210/endo.133.5.8404662

Skiadas CC, Duan S, Correll M, Rubio R, Karaca N, Ginsburg ES, Quackenbush J, and Racowsky C. 2012. Ovarian reserve status in young women is associated with altered gene expression in membrana granulosa cells. Mol Hum Reprod 18:362-371. 10.1093/molehr/gas008

Szklarczyk D, Morris JH, Cook H, Kuhn M, Wyder S, Simonovic M, Santos A, Doncheva NT, Roth A, Bork P, Jensen L, and von Mering C. 2017. The STRING database in 2017: quality-controlled proteinprotein association networks, made broadly accessible. Nucleic Acids Res 45:D362-d368. 10.1093/nar/gkw937

Peer] reviewing PDF | (2020:03:46424:2:0:NEW 1 Aug 2020) 
464

465

466

467

468

469

470

471

472

473

474

475

476

477

478

479

480

481

482

483

484

485

486

487

488

489

490
Tabibzadeh S. 1994. Cytokines and the hypothalamic-pituitary-ovarian-endometrial axis. Hum Reprod 9:947-967. 10.1093/oxfordjournals.humrep.a138621

Tesfaye D, Salilew-Wondim D, Gebremedhn S, Sohel MM, Pandey HO, Hoelker M, and Schellander K. 2016. Potential role of microRNAs in mammalian female fertility. Reprod Fertil Dev 29:8-23. $10.1071 / \mathrm{rd} 16266$

Vanacker J, Camboni A, Dath C, Van Langendonckt A, Dolmans MM, Donnez J, and Amorim CA. 2011. Enzymatic isolation of human primordial and primary ovarian follicles with Liberase $\mathrm{DH}$ : protocol for application in a clinical setting. Fertil Steril 96:379-383 e373. 10.1016/j.fertnstert.2011.05.075

Vinatier D, Dufour P, Tordjeman-Rizzi N, Prolongeau JF, Depret-Moser S, and Monnier JC. 1995. Immunological aspects of ovarian function: role of the cytokines. Eur J Obstet Gynecol Reprod Biol 63:155-168. 10.1016/0301-2115(95)02227-9

Wang W, Chen J, Luo L, Li Y, Liu J, and Zhang W. 2018. Effect of cadmium on kitl pre-mRNA alternative splicing in murine ovarian granulosa cells and its associated regulation by miRNAs. J Appl Toxicol 38:227-239. 10.1002/jat.3516

Woo I, Christenson LK, Gunewardena S, Ingles SA, Thomas S, Ahmady A, Chung K, Bendikson K, Paulson $\mathrm{R}$, and McGinnis LK. 2018. Micro-RNAs involved in cellular proliferation have altered expression profiles in granulosa of young women with diminished ovarian reserve. J Assist Reprod Genet 35:1777-1786. 10.1007/s10815-018-1239-9

Yu G, Wang LG, Han Y, and He QY. 2012. clusterProfiler: an R package for comparing biological themes among gene clusters. Omics 16:284-287. 10.1089/omi.2011.0118

Yuan S, Ortogero N, Wu Q, Zheng H, and Yan W. 2014. Murine follicular development requires oocyte DICER, but not DROSHA. Biol Reprod 91:39. 10.1095/biolreprod.114.119370

Yuan S, Wen J, Cheng J, Shen W, Zhou S, Yan W, Shen L, Luo A, and Wang S. 2016. Age-associated upregulation of EGR1 promotes granulosa cell apoptosis during follicle atresia in mice through the NF-kappaB pathway. Cell Cycle 15:2895-2905. 10.1080/15384101.2016.1208873 
Figure 1

Volcano plot of all DEGs

The orangered and blue dots represent significantly upregulated and downregulated DEGs, respectively. DEGs, differentially expressed genes; FC, fold change.

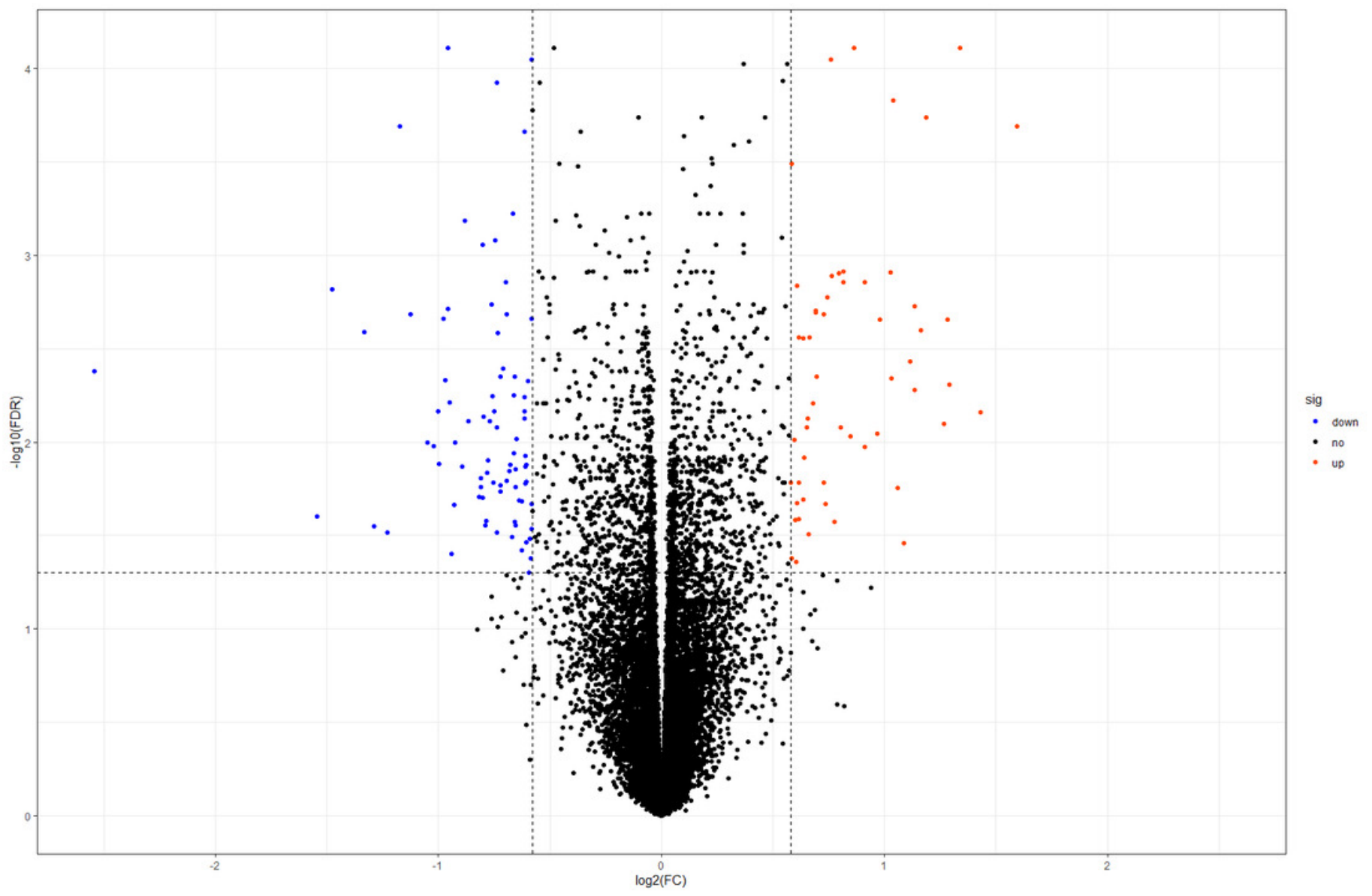




\section{Figure 2}

\section{The GO enrichment analysis of the DEGs}

\section{(A) Top 20 enriched BP terms of downregulated genes. (B) Top 20 enriched BP terms of} upregulated genes. The length of bars represents the number of genes, the color of bars represents corresponding adjusted P-value. GO, Gene Ontology; DEGs, differentially expressed genes; BP, biological process.
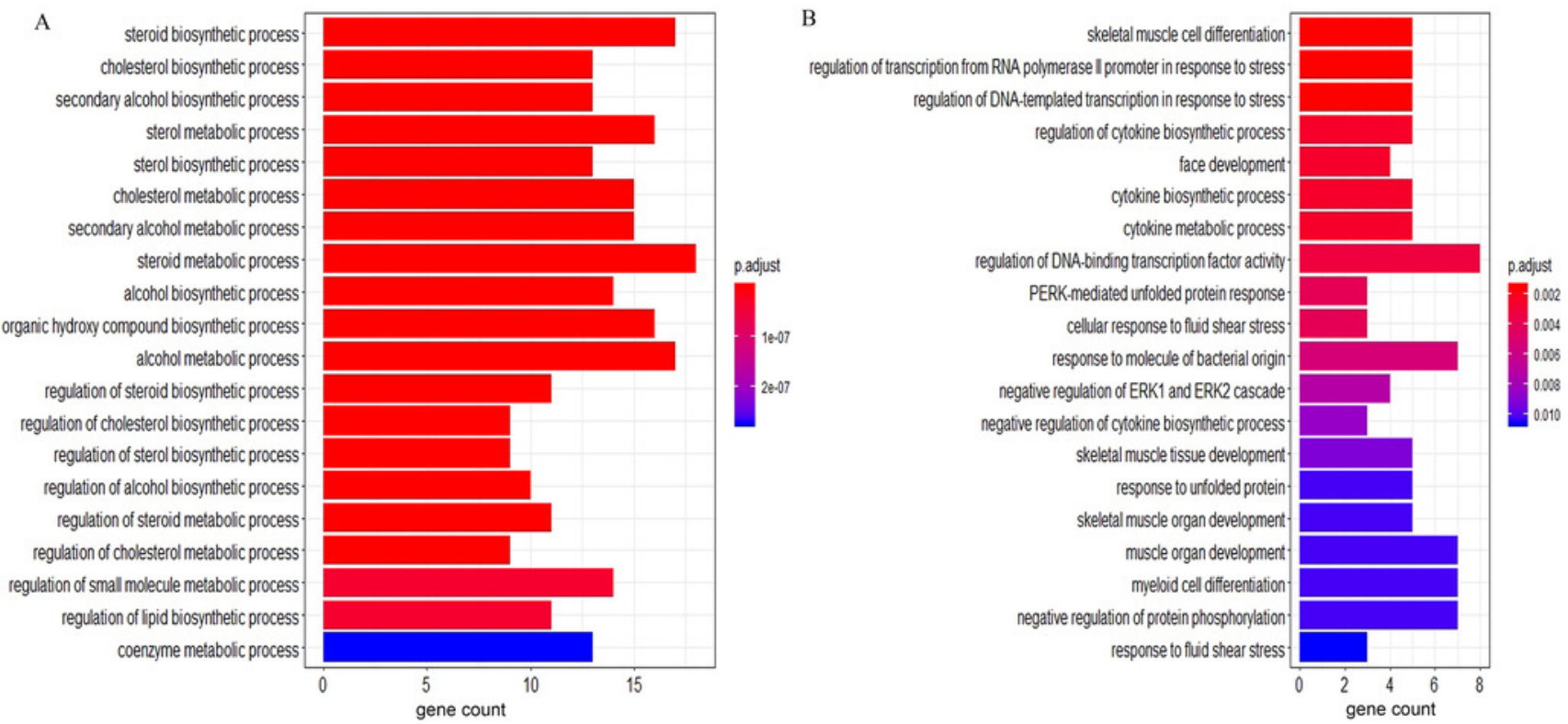
Figure 3

Pathway of steroid biosynthesis from KEGG

The genes with blue are downregulated DEGs. KEGG, Kyoto Encyclopedia of Genes and Genomes; DEGs, differentially expressed genes. 


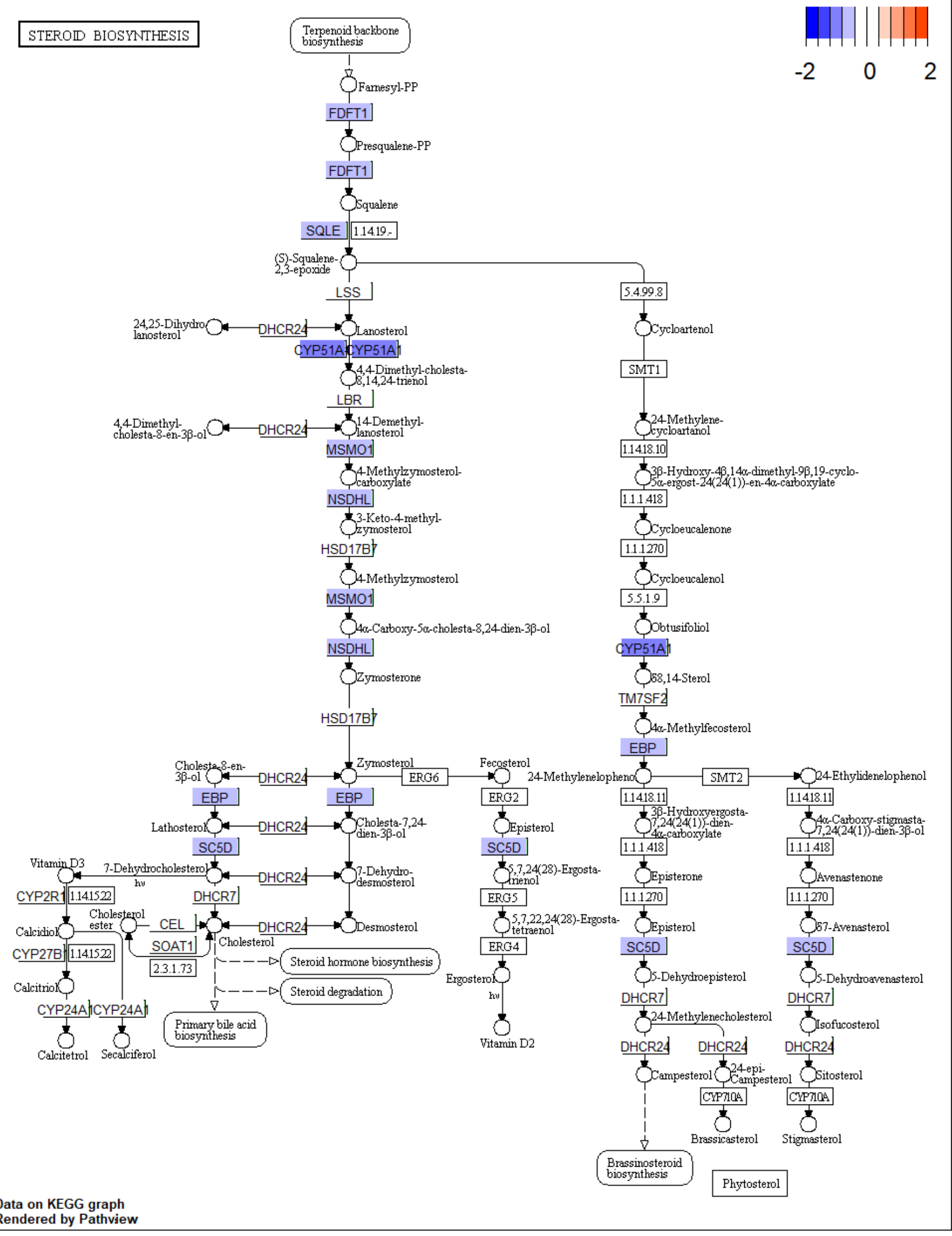


Figure 4

The PPI network and modular analysis

(A) The PPI network of DEGs. (B) module 1. (C) module 2. Node represents gene, node size represents node degree. The orangered and blue nodes represent upregulated and downregulated genes, respectively. The depth of color represents the level of differential expression and the width of lines represents the combined score between two proteins. PPI, protein-protein interaction; DEGs, differentially expressed genes.
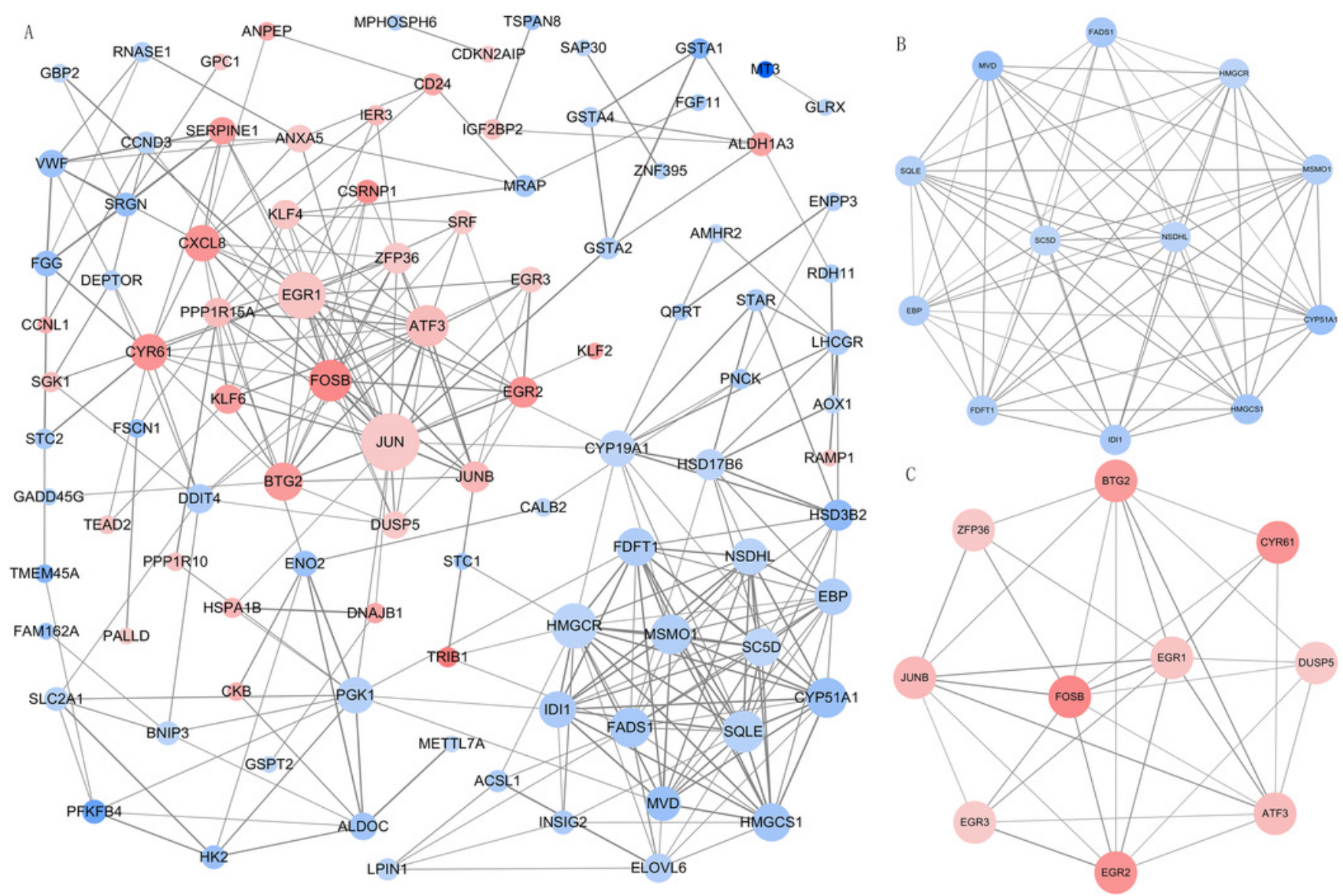
Figure 5

The DEMs-DEGs regulatory network

(A) The upregulated DEMs and targeted DEGs. (B) The downregulated DEMs and targeted DEGs. Circle nodes represent DEGs and triangle nodes represent DEMs. Orangered represent upregulation and blue represent downregulation. DEGs, differentially expressed genes; DEMs, differentially expressed miRNAs. 
A

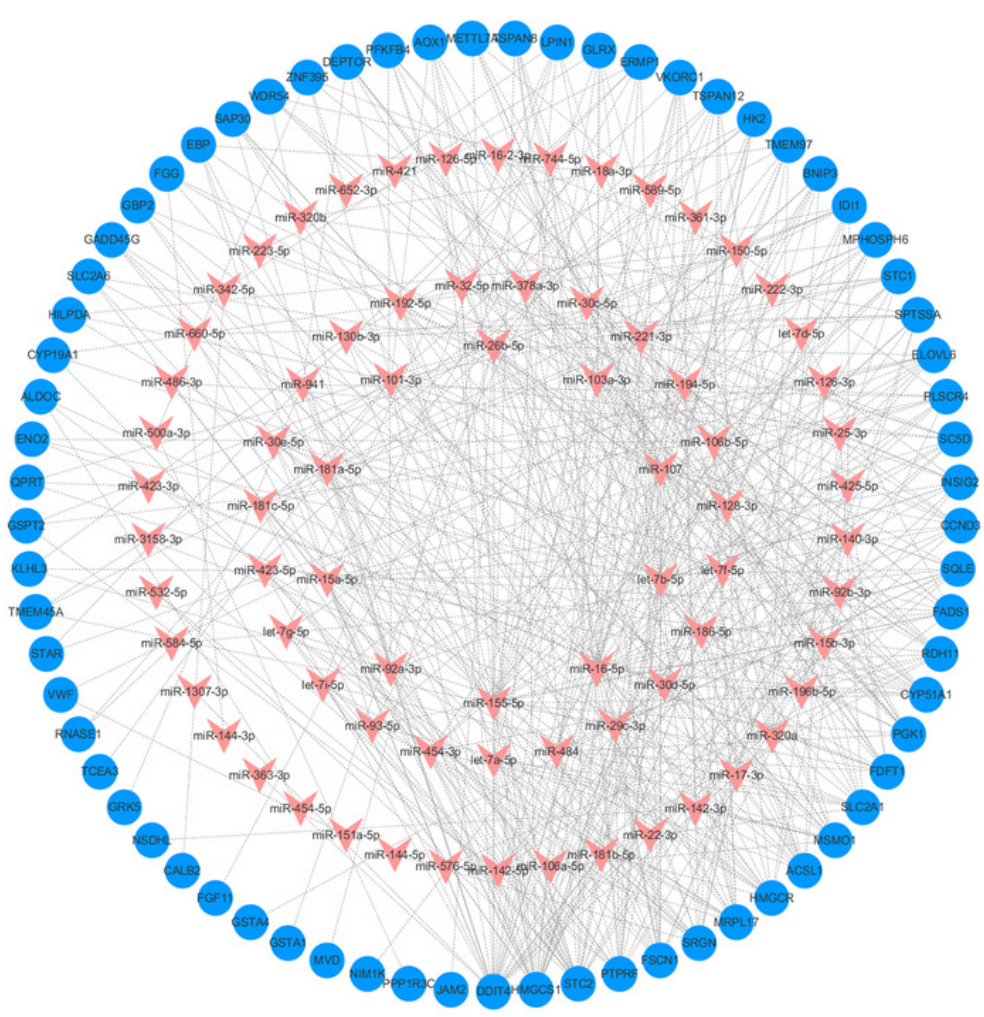

B

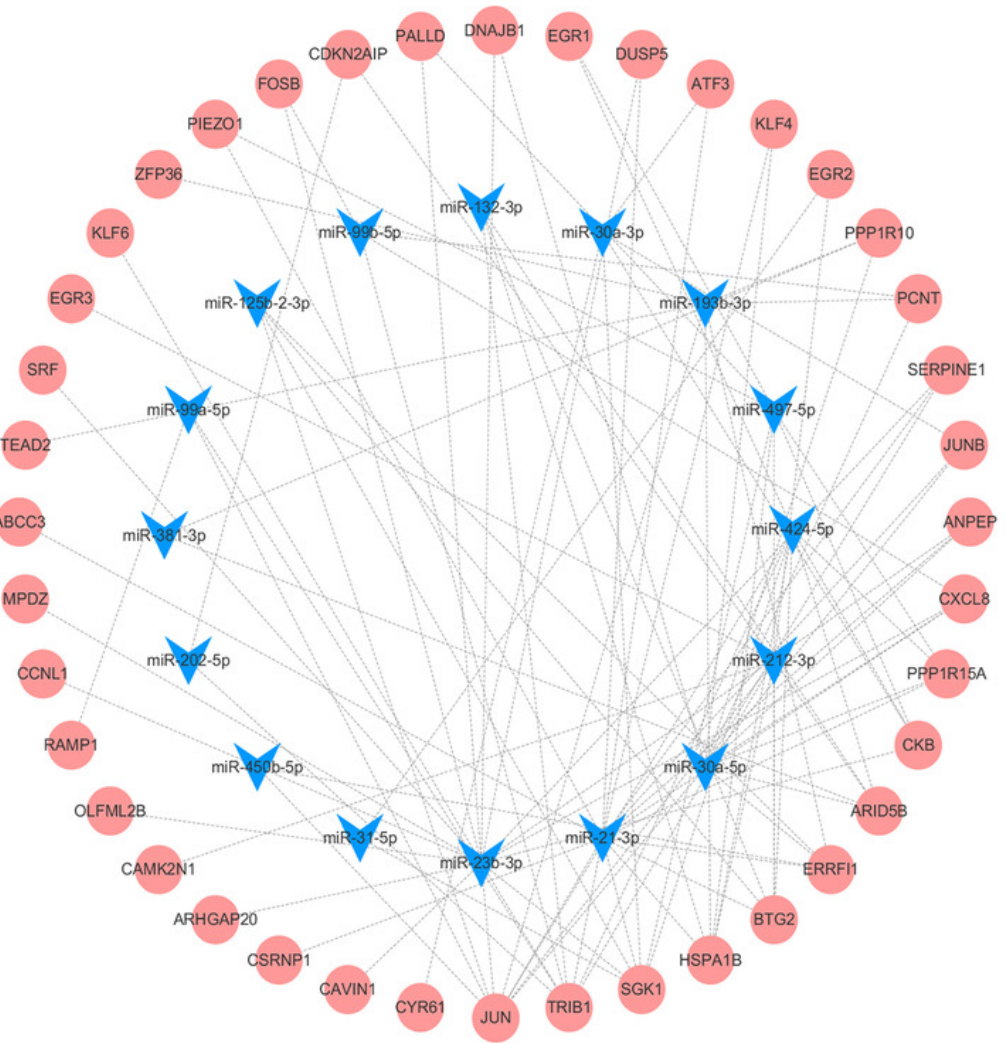


Figure 6

Result of CMap analysis

The orangered dots represent the synergistic small molecule drugs. The blue dots represent antagonistic small molecule drugs. CMap, Connectivity Map.

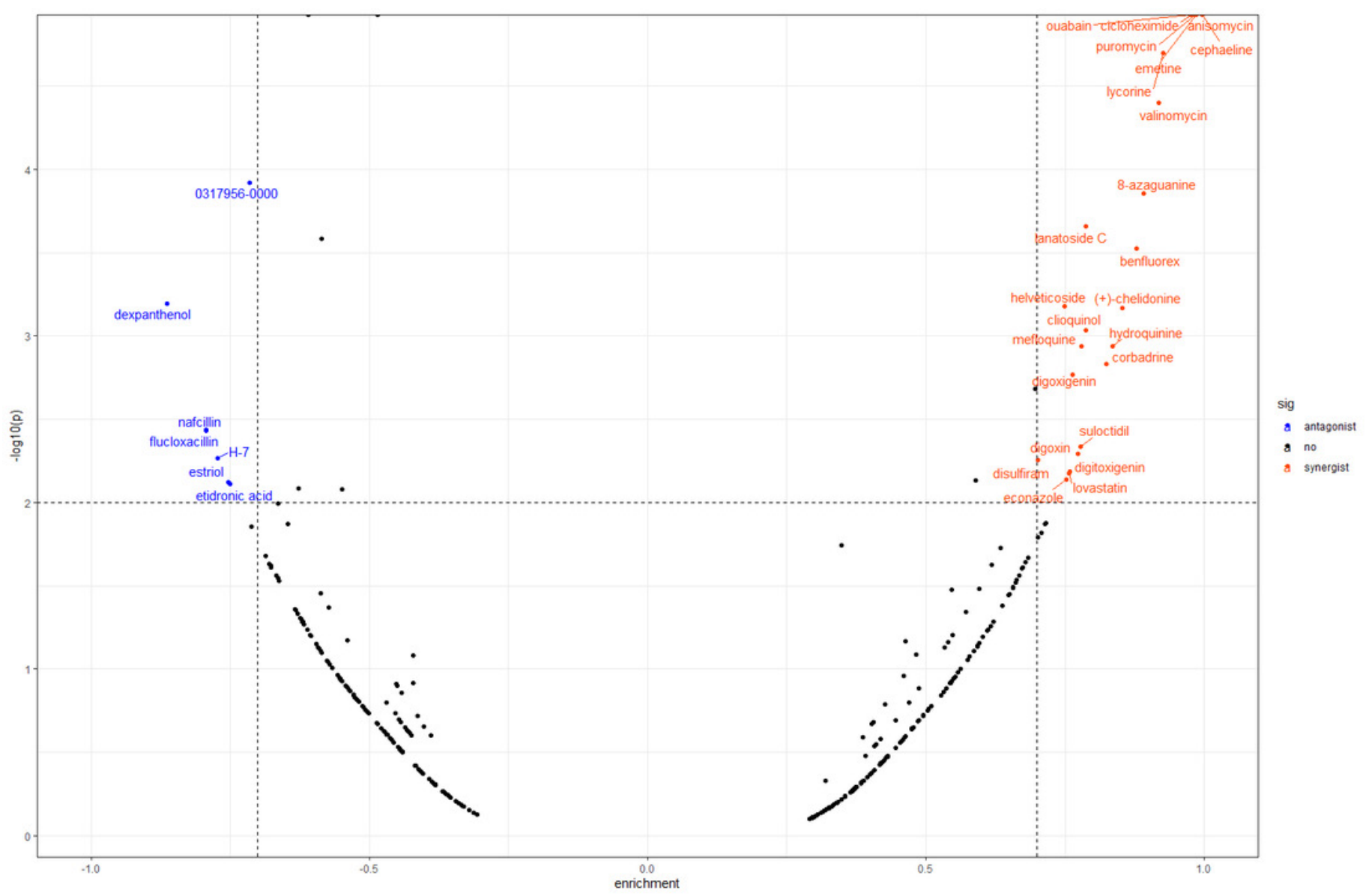




\section{Table $\mathbf{1}$ (on next page)}

Top 5 KEGG pathways of the DEGs

KEGG, Kyoto Encyclopedia of Genes and Genomes; DEGs, differentially expressed genes. 


\begin{tabular}{clllll}
\hline Pathway ID & Description & P-value & Count & Genes & State \\
\hline hsa04933 & $\begin{array}{l}\text { AGE-RAGE signaling pathway } \\
\text { in diabetic complications }\end{array}$ & 0.000389 & 4 & SERPINE1, EGR1, CXCL8, JUN & upregulated \\
hsa05166 & $\begin{array}{l}\text { Human T-cell leukemia virus 1 } \\
\text { infection }\end{array}$ & 0.000905 & 5 & EGR2, ZFP36, SERPINE1, SRF, JUN & upregulated \\
hsa05161 & Hepatitis B & 0.002359 & 4 & EGR3, EGR2, CXCL8, JUN & upregulated \\
hsa04657 & IL-17 signaling pathway & 0.004284 & 3 & FOSB, CXCL8, JUN & upregulated \\
hsa05203 & Viral carcinogenesis & 0.005122 & 4 & EGR3, EGR2, SRF, JUN & upregulated \\
hsa00100 & Steroid biosynthesis & $2.49 \mathrm{E}-11$ & 7 & CYP51A1, FDFT1, MSMO1, EBP, NSDHL, & downregulated \\
hsa00900 & Terpenoid backbone & $1.39 \mathrm{E}-05$ & 4 & SC5D, SQLE & downregulated \\
hsa04913 & Oviosynthesis & 0.00035 & 4 & LHCGR, STAR, HSD3B2,CYP19A1 & downregulated \\
hsa04066 & HIF-1 signaling pathway & 0.000908 & 5 & HK2, PGK1, ALDOC, ENO2, SLC2A1 & downregulated \\
hsa00010 & Glycolysis / Gluconeogenesis & 0.001222 & 4 & HK2, PGK1, ALDOC, ENO2, & downregulated \\
\hline
\end{tabular}

1 


\section{Table 2 (on next page)}

Enriched GO BP terms (top 5) and significantly enriched KEGG pathways of genes in the top two modules

GO, Gene Ontology; BP, biological process; KEGG, Kyoto Encyclopedia of Genes and Genomes. 


\begin{tabular}{|c|c|c|c|c|}
\hline Modules & & Description & P.adjust & Count \\
\hline \multirow[t]{7}{*}{ module 1} & BP terms & cholesterol biosynthetic process & $3.53 \mathrm{E}-24$ & 11 \\
\hline & & secondary alcohol biosynthetic process & $3.53 \mathrm{E}-24$ & 11 \\
\hline & & sterol biosynthetic process & 4.99E-24 & 11 \\
\hline & & cholesterol metabolic process & $2.88 \mathrm{E}-21$ & 11 \\
\hline & & secondary alcohol metabolic process & $3.39 \mathrm{E}-21$ & 11 \\
\hline & KEGG pathway & Steroid biosynthesis & $1.02 \mathrm{E}-15$ & 7 \\
\hline & & Terpenoid backbone biosynthesis & $1.09 \mathrm{E}-07$ & 4 \\
\hline \multirow[t]{8}{*}{ module 2} & BP terms & skeletal muscle cell differentiation & $2.30 \mathrm{E}-05$ & 4 \\
\hline & & muscle organ development & 0.00019411 & 5 \\
\hline & & skeletal muscle tissue development & 0.00019411 & 4 \\
\hline & & skeletal muscle organ development & 0.00019411 & 4 \\
\hline & & regulation of nuclear-transcribed mRNA poly(A) tail shortening & 0.001616513 & 2 \\
\hline & KEGG pathway & Human T-cell leukemia virus 1 infection & 0.019978459 & 3 \\
\hline & & C-type lectin receptor signaling pathway & 0.043059356 & 2 \\
\hline & & Osteoclast differentiation & 0.043059356 & 2 \\
\hline
\end{tabular}

1 


\section{Table 3(on next page)}

LC-MS/MS detected steroids that varied in GCs of DOR with significant difference

LC-MS/MS, liquid chromatography-tandem mass spectrometry; GCs, granulosa cells; DOR, diminished ovarian reserve; FC, fold change. 


\begin{tabular}{|c|c|c|c|c|c|c|c|}
\hline Components & Formula & $\mathrm{m} / \mathrm{z}$ & $\mathrm{FC}$ & p-value & Class & Sub class & label \\
\hline Hydroxyprogesterone & $\mathrm{C}_{21} \mathrm{H}_{30} \mathrm{O}_{3}$ & 330.2190 & 0.0404 & 0.0381 & $\begin{array}{l}\text { Steroids and } \\
\text { steroid } \\
\text { derivatives }\end{array}$ & $\begin{array}{l}\text { Pregnane } \\
\text { steroids }\end{array}$ & down \\
\hline Progesterone & $\mathrm{C}_{21} \mathrm{H}_{30} \mathrm{O}_{2}$ & 314.2244 & 0.0785 & 0.0025 & $\begin{array}{l}\text { Steroids and } \\
\text { steroid } \\
\text { derivatives }\end{array}$ & $\begin{array}{l}\text { Pregnane } \\
\text { steroids }\end{array}$ & down \\
\hline 3 alpha-hydroxydesogestrel & $\mathrm{C}_{22} \mathrm{H}_{30} \mathrm{O}_{2}$ & 326.2241 & 0.0657 & 0.0027 & $\begin{array}{l}\text { Steroids and } \\
\text { steroid } \\
\text { derivatives }\end{array}$ & Estrane steroids & down \\
\hline $\begin{array}{l}\text { (6beta,8xi,11beta,14xi,16alpha)-9- } \\
\text { fluoro-6,11,17,21-tetrahydroxy-16- } \\
\text { methylpregna-1,4-diene-3,20-dione }\end{array}$ & $\mathrm{C}_{22} \mathrm{H}_{29} \mathrm{FO}_{6}$ & 408.1960 & 0.0290 & 0.0093 & $\begin{array}{l}\text { Steroids and } \\
\text { steroid } \\
\text { derivatives }\end{array}$ & Hydroxysteroids & down \\
\hline $\begin{array}{l}\text { (6beta,8xi,11beta,14xi,16alpha)-9- } \\
\text { fluoro-6,11,17,21-tetrahydroxy-16- } \\
\text { methylpregna-1,4-diene-3,20-dione }\end{array}$ & $\mathrm{C}_{22} \mathrm{H}_{29} \mathrm{FO}_{6}$ & 408.1959 & 0.0340 & 0.0179 & $\begin{array}{l}\text { Steroids and } \\
\text { steroid } \\
\text { derivatives }\end{array}$ & Hydroxysteroids & down \\
\hline 4,6-cholestadien-3-one & $\mathrm{C}_{27} \mathrm{H}_{42} \mathrm{O}$ & 382.3229 & 0.0343 & 0.0352 & $\begin{array}{l}\text { Steroids and } \\
\text { steroid } \\
\text { derivatives }\end{array}$ & $\begin{array}{l}\text { Cholestane } \\
\text { steroids }\end{array}$ & down \\
\hline Cholest-4-en-3-one & $\mathrm{C}_{27} \mathrm{H}_{44} \mathrm{O}$ & 384.3385 & 0.0796 & 0.0273 & $\begin{array}{l}\text { Steroids and } \\
\text { steroid } \\
\text { derivatives }\end{array}$ & $\begin{array}{l}\text { Cholestane } \\
\text { steroids }\end{array}$ & down \\
\hline
\end{tabular}

